\title{
La Motivación como Estrategia Educativa Empresarial para Impulsar la Creación de Empresas Socialmente Responsables en los Estudiantes de Educación Superior de Instituciones Públicas
}

\author{
María Lourdes Atondo Soto ${ }^{1}$ \\ Martha Elena Sánchez Soto ${ }^{2}$ \\ Ivonne Esmeralda Lizárraga Coronado ${ }^{3}$
}

MOTIVATION AS AN ENTERPRISE EDUCATION STRATEGY TO PROMOTE THE CREATION OF

SOCIALLY RESPONSIBLE COMPANIES IN HIGHER EDUCATION STUDENTS OF PUBLIC INSTITUTIONS

\section{Resumen}

Las instituciones públicas de educación superior, tienen el compromiso de formar profesionistas, comprometidos, con un alto sentido humano y de competencia, capaces de crear, desarrollar e innovar; con visión hacia el desarrollo sustentable, tecnológico, social y económico que demanda el entorno globalizado.

Por consiguiente, en la presente investigación etnográfica se identificaron, las áreas de oportunidad del estudiante que lo conduzca a desarrollar una cultura empresarial y emprenda su propio proyecto, detectando, las herramientas motivacionales que deben estimular su interés de innovador, en las diferentes carreras que ofrece las instituciones públicas, logrando involucrar un objetivo común. Todo lo anterior encaminado hacia su vocación empresarial.

Con los resultados se busca identificar los factores internos y externos, dándole confianza en sí mismo, para impulsar su propia empresa, estableciendo un compromiso, con su familia, amigos y el entorno, que lo impulse a participar en un contexto empresarial socialmente responsable.

Palabras clave: Motivación, educación, emprender, empresa, Socialmente responsable, instituciones públicas, cultura empresarial, medio ambiente.

\section{Abstract}

The public institutions of higher education have the commitment to train professionals, committed, with a high human sense and competence, capable of creating, developing and innovating; with a vision towards the sustainable, technological, social and economic development that the globalized environment demands. Therefore, in the present ethnographic research they were identified, the areas of opportunity of the student that leads him to develop a business culture and undertake his own project, detecting, the motivational tools that should stimulate his interest as an innovator, in the different careers offered public institutions, managing to involve a common objective. All the above directed towards his business vocation. The results seek to identify the internal and external factors, giving him self-confidence, to boost his own company, establishing a commitment, with his family, friends and the environment, that drives him to participate in a socially responsible business context.

Keywords: Motivation, education, entrepreneurship, company, Socially responsible, public institutions, corporate culture, environment.

\footnotetext{
${ }^{1}$ María Lourdes Atondo Soto. Maestra de tiempo completo en Instituto Tecnológico de Hermosillo. Maestra de Asignatura en la Universidad de Sonora. Jefe del Departamento de Ciencias Económico Administrativo en el Instituto Tecnológico de Hermosillo. Doctorado en Administración Educativa. Integrante de la academia de Administración.

${ }^{2}$ Martha Elena Sánchez Soto. Maestra de tiempo completo del Instituto Tecnológico de Hermosillo. Maestra en Administración.

${ }^{3}$ Ivonne Esmeralda Lizárraga Coronado. Maestra de tiempo completo del Instituto Tecnológico de Hermosillo. Jefe de la división de Estudios Superiores. Maestra en Administración.
} 


\section{Introducción}

La transformación de la educación superior, considera, que la formación integral se fundamenta en el desarrollo de un cumulo de conocimientos teóricos y prácticos, estrategias de aprendizaje, habilidades, capacidades y el fortalecimiento de la cultura social del medio ambiente, enriqueciendo y favoreciendo con ello la trayectoria académica.

Sin embargo, la condición humana que define al individuo, identifica las oportunidades, permitiéndole trazar un rumbo en los proyectos que desea realizar. Para realizar estos, la razón de ser debe de convertir los hechos en realidades, mediante el compromiso de cumplir con planes académicos, fundamentando cada uno de ellos en el aprendizaje durante su formación profesional.

La formación educativa es la herramienta que emana del conocimiento legítimo para favorecer al estudiante en su quehacer diario durante su desarrollo profesional. Contribuyendo con ello al desarrollo social y cultural del medio en que se desenvuelve.

Es definitivo, que el aprendizaje está: En el aula de clases, interactuando con las personas y maestros que imparten el conocimiento y los motivan a realizarse como personas de éxito, mostrando diferentes estrategias, que van desde la actitud, hábitos, habilidades, destrezas indispensables para la vida y el desarrollo del pensamiento crítico y científico.

La educación y la formación para impulsar la necesidad de desarrollar estrategias empresariales socialmente responsables, comienza precisamente en la persona, enfatizando en la autonomía interior de su propio conocimiento, siendo protagonista de su adecuado aprendizaje.

La educación es esencial para adquirir y reforzar la motivación educativa, favoreciendo al individuo en su crecimiento y permitiendo la transformación en la sociedad, siendo esto definitivo para la obtención de conocimiento, formando hombres comprometidos para el entorno: político, cultural, social, ambiental y económico.

Por consiguiente, hacer mención de la motivación como Estrategia Educativa es imperativo, la situación actual de las Instituciones de Educación Superior Pública, se encuentran inmersas en la desmotivación del estudiante. Es conocido que él hombre, debe basarse en su propia motivación y buscar mecanismos de defensa para lograr cumplir sus expectativas y reforzarse durante su estancia en el Sistema Educativo.

Es importante entonces, que las exigencias de una sociedad en la actualidad, es qué la educación superior ofrezca al ambiente empresarial, profesionistas motivados a innovar y crear empresas socialmente responsables que satisfagan y cubran las necesidades del hombre y su entorno.

La Motivación como Estrategia Educativa Empresarial, es un proceso en la búsqueda de motivos que inspiren al hombre a generar acciones innovadoras para lograr un resultado deseado, garantizando el desarrollo personal, comprometiendo a las instituciones de educación superior a gestionar actividades extracurriculares encaminadas al profesionalismo de sus estudiantes, en el proceso de enseñanza aprendizaje.

Se tiene entonces como objetivo, el plantear estrategias motivacionales para impulsar la creación de empresas proporcionando con ello las herramientas necesarias para el desarrollo de empresas socialmente responsables. De tal manera que este programa parte de la premisa de que las instituciones de educación superior deben guiar a los estudiantes con sus ideas hasta la creación de su propia empresa socialmente responsable. 


\section{Marco Conceptual}

La creatividad, es relevante en el ser humano, porque esta le permite en un momento dado estar en una realidad e imaginarla y cuando esto sucede entra en un proceso de análisis, ordenando sus ideas, para posteriormente realizar con esto, una planeación de un modelo mental a través de una pantalla imaginaria visualizando anticipadamente su futuro, proporcionando con esto la base para una acción efectiva que dará como resultado la habilidad para administrar sus ideas. Según (Ezequiel Ander- Egg), la planeación es un faro, guía, tiene una luz a la que se dirige a alguna cosa, tiene orientación, la planeación es la brújula que no deja que se pierda el camino.

Por lo que, cuando se habla de la formalidad de una iniciativa para emprender una empresa socialmente responsable, el emprendedor de negocios, debe de resaltar que debe de autoevaluarse para ubicarse emocionalmente, ¿qué es lo que quiere emprender?, para transformar esas ideas en acciones que beneficien a una sociedad carente de sustentabilidad. Y llevar a cabo su planeación de trabajo, bajo ese modelo de disciplina, para lograr así el éxito deseado.

El planear-emprender, encierra muchos desafíos que debe vencer el ser humano en el proceso de enseñanzaaprendizaje, debe de tener claro en su mente que no nada más es la Técnica la que debe de desarrollar, sino también debe de existir el enamoramiento, la pasión por lo que quiere hacer y cuando sea necesario, reorientar las actividades planeadas e ir implantando más ideas nuevas para este semillero, convirtiendo estas en oportunidades de negocios. Dicho de otra forma, el modelo de negocios no nada más debe de encerrar una metodología de actividades abstractas, también debe incluir las emociones de las personas.

El individuo por naturaleza se identifica con el quién soy, y este es un paso importante en la vida del mismo, esclarecerá, los dos puntos siguientes:
- Definir qué es lo que desea realizar.

- Razones que tiene para hacerlo.

Una vez hecho su introspección y reconociendo sus habilidades personales, se pretende que el estudiante tenga una visión más clara de lo que quiere y hacia dónde va dirigir su esfuerzo. El tener tal claridad es una característica de las personas que son emprendedoras. Además que no tienen ningún prejuicio de trabajar con personas que los lleven a culminar su sueño.

El estudiante universitario debe de estar preparado para enfrentar toda aquella situación que se derive del accionar y de su razón de ser, además transformar sus ideas y gestionar el trabajo en equipo con los involucrados en su quehacer diario. Hace mención Balderrama (2010) en su análisis, el gran reto de la gestión de equipos en el mundo actual es encontrar qué motiva a cada individuo para lograr el objetivo, ya sea personal o del equipo para impulsar la creación de la empresa socialmente responsables.

Asimismo, el entorno que rodea al emprendedor y la influencia que se tiene sobre él, es muy importante, ya que tenemos en primer plano, la familia y amigos, que dependiendo del contexto, le dan fuerza a la actividad de emprender, pero de igual manera, pueden influir de manera negativa. Existe la otra parte fundamental, que son, los factores sociales, económicos, políticos y culturales, estos, son los que establecen las oportunidades y las amenazas en el entorno en el que el emprendedor opera.

Contextualmente, la gestión del espíritu empresarial en las instituciones de educación superior es una labor compleja y sutil, cuyos ingredientes imprescindibles, son el saber identificar, allí donde se encuentre, captarlo, desarrollarlo y retenerlo. Hay Instituciones que son excelentes captadoras de talento pero después no consiguen desarrollarlo o retenerlo. Por ello resulta cada vez más valioso el papel de un liderazgo capaz de equilibrar todas estas tareas relacionadas con el talento humano, para que las organizaciones y las personas puedan dar lo mejor de sí. (Pilar Jericó) 
Se empezará por definir: Espíritu Empresarial desde una perspectiva más general, La Comisión de las Comunidades Europeas, la define como una forma de pensar o una mentalidad que incluye la motivación y la capacidad del individuo, bien sea de forma independiente o dentro de una organización, para identificar una oportunidad y luchar por ella, y así producir nuevo valor económico y/o social. La iniciativa emprendedora incluye la propensión a inducir cambios en uno mismo, la capacidad de aceptar y apoyar la innovación provocada por factores externos, de dar la bienvenida al cambio, de asumir la responsabilidad por las propias acciones (sean positivas o negativas), de terminar lo que se empieza, de saber en qué dirección se está yendo, de establecer objetivos y cumplirlos, y de tener la motivación necesaria para el éxito.

Seguido a esto existe una gran responsabilidad para el futuro profesionista y empresario, en el esclarecimiento de empresas socialmente responsables, donde tenemos entonces, que es todo aquello que contribuye activamente y voluntariamente para mejorar el entorno social, económico y ambiental, en donde busca una ventaja competitiva y un valor agregado.

Aunado a lo anterior, la iniciativa emprendedora es primordial en todos los ámbitos de la sociedad, es fundamentalmente significativa entre los jóvenes, en donde es caracterizada por su creatividad, su innovación, su espíritu emprendedor y aventurero, su menor aversión al riesgo y mayor sensibilidad, y su concientización hacia los cambios que emanen al desarrollo del entorno en que se encuentren.

En relación a la motivación, tenemos que es un motor necesario para el desarrollo adquiriendo con ello, un compromiso en diversas actividades y acciones que permitan al individuo $\mathrm{y}$ en este caso al alumno, mantener una actitud positiva hacia el trabajo empresarial.

La motivación como estrategia para impulsar al estudiante, representa una pieza fundamental durante la formación académica en las instituciones de educación superior, implicando desarrollar una formación profesional en el proceso de enseñanza-aprendizaje del alumno, resultando de ello una cultura empresarial, y que posteriormente pueda ser aplicado en la empresa socialmente responsable, como un compromiso de los jóvenes en contribuir con su entorno y también brindar una ventaja competitiva a su propuesta empresarial.

Por consiguiente, el carecer de programas de cultura empresarial para desarrollar empresas socialmente responsables, limita al estudiante su desarrollo durante el proceso de formación profesional.

Entonces tenemos que la cultura empresarial involucra el espíritu empresarial y la iniciativa emprendedora que debe tener el estudiante como reto para buscar una sociedad mejor.

\subsection{Planteamiento del Problema}

Durante la formación profesional otorgada en las instituciones públicas, existe en el alumno, deficiencias en cuanto al desarrollo de acciones motivacionales enfocadas a fomentar e impulsar al alumno hacia la creación de empresas socialmente responsables, así como también, bajo nivel de orientación de la creatividad e innovación de las ideas, promoviendo así el interés en los estudiantes e identificar sus fortalezas creativas y poderlas adaptar a los requerimientos de la actividad empresarial. Por lo tanto se considera que la problemática actual en la institución es la falta de motivación del estudiante hacia programas académicos encaminados a la cultura empresarial socialmente responsable $y$ actividades motivacionales extracurriculares que lleven al estudiante a analizar los mecanismos que los motive a emprender su empresa.

\subsection{Objetivo General}

Plantear Estrategias Motivacionales que impulse la Creación de Empresas Socialmente Responsables en los Estudiantes de Instituciones Públicas de Educación Superior. 


\subsubsection{Objetivos Específicos}

- Desarrollo de un programa extracurricular que impulse la creación de empresas socialmente responsables.

- Talleres de talento emprendedor enfocados a la actitud emprendedora para la creación de Empresas Socialmente Responsables.

- Círculos motivacionales de negocios.

\section{Metodología}

El método que se utilizó para la elaboración de la presente investigación es el etnográfico y la metodología a seguir es la descriptiva-explicativa, permitiendo con ello identificar las características que motivan a los estudiantes hacia una cultura emprendedora para la creación de su propia empresa socialmente sustentable.

\subsection{Universo de la Investigación}

Para el universo se tomaron en cuenta la matricula del semestre impar (2015-1) de 3618 alumnos, en esas fechas cada año se realiza la preparación de estudiantes para el Encuentro Nacional Estudiantil de los Institutos Tecnológicos en su fase local. Se invita a todos los alumnos a participar con sus ideas a formar su propia empresa.

\subsection{Análisis de los Resultados}

El total de alumnos que tienen las 6 carreras que asistieron a la actividad académica son 2139 estudiantes, correspondiendo el 77.38 del universo 3618 en ese periodo, como se observan en el cuadro 1.

Asistentes a la actividad académica para Impulsar la Creación de Empresas Socialmente Responsable de 2139 fueron 170 estudiantes correspondiendo el 63. 89 porciento, como se observa en el cuadro 2.

Cuadro No. 1 Porcentaje de alumnos inscritos 2015-1

\begin{tabular}{|l|l|l|}
\hline CARRERAS & $\begin{array}{c}\text { No. Alumnos inscritos en el } \\
\text { escolarizado. }\end{array}$ & $\%$ alumnos de la matricula \\
\hline Administración & 164 & $4.45 \%$ \\
\hline Ing. Gestión empresarial & 409 & $11.10 \%$ \\
\hline Lic. Informática & 102 & $2.7 \%$ \\
\hline Ing. Sistemas & 333 & 9.02 \\
\hline Ing. Electrónica & 421 & $11.42 \%$ \\
\hline Ing. Industrial & 710 & 38.69 \\
\hline Total & 2139 & 77.38 \\
\hline
\end{tabular}

Fuente: Elaboración propia 
Cuadro No. 2 Asistencia actividad académica 2015-1

\begin{tabular}{|l|l|l|}
\hline CARRERAS & $\begin{array}{c}\text { No. Alumnos que asistieron } \\
\text { a las actividades académicas. }\end{array}$ & $\begin{array}{c}\% \text { alumnos asistentes a las } \\
\text { actividades académicas. }\end{array}$ \\
\hline Administración & 13 & 4.9 \\
\hline Ing. Gestión empresarial & 67 & 16.38 \\
\hline Lic. Informática & 26 & 25.49 \\
\hline Ing. Sistemas & 38 & 11.14 \\
\hline Ing. Electrónica & 24 & 5.7 \\
\hline Ing. Industrial & 2 & 0.28 \\
\hline Total & 170 & 63.89 \\
\hline
\end{tabular}

Fuente: Elaboración propia

Considerando el número de alumnos 3618 en el periodo 2015-1 (100\%) solo 170 estudiantes son los que les intereso asistir a la actividad académica organizada por el área de vinculación.

\subsection{Desarrollo del Tema}

La responsabilidad social empresarial, se ha convertido en una forma de gestionar y hacer negocios, ocupándose para ello la propia empresa, buscando ser sustentable en lo económico, social y ambiental. Con ello busca que tanto las generaciones futuras de empresas sean sustentables y los empresarios emprendedores busquen la preservación del medio ambiente.

Actualmente, es posible encontrar programas de responsabilidad social en el cuidado del ambiente en prácticamente todas las principales economías del mundo, así como también en muchos países en desarrollo como por ejemplo: China y México entre otros.
Las instituciones de educación superior públicas tienen el compromiso de promover el impulso entre los estudiantes de crear y hacer crecer empresas jóvenes, en donde se les motive a identificar su propio modelo de negocios, proporcionándole, actividades extracurriculares que los guíen y encamine a la sustentabilidad como es el programa: Espíritu emprendedor ético, sustentable. Así como brindarle un seguimiento estricto para observar el cumplimiento de sus metas y objetivos planteados durante los talleres del programa. Ofreciendo también, un espacio físico en sus etapas de inicio, mientras adquiere mayor formalidad como empresario socialmente responsable.

Por otra parte, se debe tener en cuenta por parte de los estudiantes como futuros empresarios, que lo que les ofrecen las instituciones de educación superior públicas, con la implementación de este programa es una guía que sirva como oportunidad para enfrentar una vida empresarial de forma estrictamente fuerte, a través de la capacitación mediante los talleres y asesoramiento individualizado continuo. 
La Motivación como Estrategia Educativa Empresarial para impulsar la creación de empresas socialmente responsable en estudiantes de Educación Superior de Instituciones Públicas. Se debe de promover a través del programa espíritu emprendedor ético, sustentable, con la mentalidad que es un área de oportunidad para el impulso de empresas nuevas ya sea pequeñas o medianas de los estudiantes.

La estrategia de negocios que impulse a seguir a los estudiantes en su creación de empresas, debe estar enfocada a la responsabilidad social, convirtiéndola en su ventaja competitiva.

Con la creación de empresas comprometidas socialmente responsables, se espera un cambio positivo en la sociedad, en donde la responsabilidad social empresarial, es un motivo importante en el estudiante, para generar empresas competitivas.

\subsubsection{Ventajas al planear estrategias motivacionales encaminadas a la sustentabilidad en la creación de empresas}

- Incrementa la competitividad en el entorno en empresas de ese nivel.

- Aumente la posibilidad de la creación de empresas promovidas por las instituciones de educación superior públicas.

- Los índices de fracasar al establecer su propia empresa serían menores, que en otras circunstancias.

- El programa a implementar es una garantía para incrementar el número de empresas creadas por estudiantes durante su formación profesional.

- Con este programa se puede promocionar estrategias de asociación y clústeres como una forma de desarrollo empresarial.

\section{Resultados}

Las instituciones de educación superior deben de preparar a sus estudiantes para los cambios de política educativa que emanan del gobierno, tanto federal como estatal. Quedando claro que esa política educativa trae consigo cambios en programas académicos y es donde se debe de aprovechar para enunciar, describir y desarrollar una cultura empresarial socialmente responsable.

Por consiguiente, de acuerdo a las observaciones y como resultado, se presenta un diagnostico minucioso, en donde tenemos lo siguiente:

- Existe una desorientación por parte de las Institución hacia los alumnos, en relación a su motivación y formación de una Empresa Socialmente Responsable.

- No se realiza una promoción intensiva para participar en los eventos académicos de motivación hacia una cultura empresarial socialmente responsable.

- El académico no le interesa involucrarse con los estudiantes para impulsarlos a que tengan una mente emprendedora.

- No existen programas estructurados extra-clase continuos que los lleve a identificar su vocación empresarial socialmente responsable.

- El estudiante como ser humano necesita orientación hacia qué camino seguir y es donde la institución debe impulsarlos y guiarlos hacia el bien común en una sociedad con diversas necesidades. 


\section{Propuesta}

Cuadro No. 3 Propuesta de un programa de espíritu emprendedor sustentable

\begin{tabular}{|c|c|}
\hline Propuesta: & $\begin{array}{l}\text { Programa: Espíritu emprendedor Ético, sustentable en la Educación Superior para } \\
\text { Instituciones Públicas. }\end{array}$ \\
\hline Duración: & 20 horas \\
\hline Impartido: & Sexto semestre \\
\hline Objetivo: & Desarrollar las capacidades de los estudiantes, mediante dinámicas motivacionales. \\
\hline Tema 1 & $\begin{array}{l}\text { Taller "Emprender una Cultura Socialmente Responsable” } \\
\text { Objetivo: Pensar, sentir, actuar y orientar hacia la creación de la riqueza ambiental, } \\
\text { aprovechando oportunidades del entorno. }\end{array}$ \\
\hline Tema 2 & $\begin{array}{l}\text { Taller “Conócete a ti mismo y lograr lo que deseas con éxito” } \\
\text { Objetivo: Detallar sus habilidades personales para conocer, el cómo quiero que me } \\
\text { vean. El yo-Empresa. }\end{array}$ \\
\hline Tema 3 & $\begin{array}{l}\text { Taller motivacional emprendedor para la creación de Empresas Socialmente } \\
\text { Responsables. } \\
\text { Objetivo: Determinar la actitud y obtener una respuesta diferente a la hora de } \\
\text { desarrollar la actividad empresarial socialmente responsable. }\end{array}$ \\
\hline Tema 4 & $\begin{array}{l}\text { Taller "Líderes de Negocios" } \\
\text { Objetivo: Desarrollar una visión global ambiental, cuyo resultado es la creación de } \\
\text { valor que beneficie al emprendedor, la economía y la sociedad. }\end{array}$ \\
\hline Tema 5 & $\begin{array}{l}\text { Taller "Identifica el mejor Modelo de Negocios" } \\
\text { Objetivo: Analizar modelos de negocios a través de casos prácticos en empresas } \\
\text { establecidas. }\end{array}$ \\
\hline Tema 6 & $\begin{array}{l}\text { Taller “Círculos motivacionales de Negocios” } \\
\text { Objetivo: idear, planear, accionar y ajustar negocios mediante estrategias sustentables. }\end{array}$ \\
\hline Tema 7 & $\begin{array}{l}\text { Taller “Seguimiento de un Modelo de Negocios” } \\
\text { Objetivo: Guiar a la actualizar vanguardista de acuerdo a los hechos que se presenten. }\end{array}$ \\
\hline
\end{tabular}

Fuente: Elaboración propia 


\section{Conclusiones}

Se concluye que las instituciones de educación superior públicas deben de detallar, el sentir, la actitud, los pensamientos y comportamientos del estudiante para una cultura empresarial socialmente sustentable y así mejorar e implementar cada día nuevas estrategias que permitan con ello que el alumno se motive a emprender y ser competitivo durante su formación profesional.

El programa espíritu emprendedor ético, sustentable en la educación superior para instituciones públicas, es una alternativa para que los estudiantes se motiven a desarrollar empresas socialmente sustentables, en donde también se obtendrá un desarrollo profesional, un cumulo de empresas formadas por los estudiantes, permitiendo la vinculación a la institución con los diferentes sectores, lograr objetivos conjuntos institución-empresa.

El promocionar el programa permitirá involucrar a todos los actores (alumnos, institución, maestros, directivos) para lograr un desempeño empresarial exitoso.

También este programa resuelve la problemática planteada en la investigación, debido a que se identifica la actitud y la iniciativa a la cultura empresarial al emprender un negocio socialmente sustentable por el estudiante.

Por otra parte también, el alumno debe ser atraído al mundo empresarial y quedarle claro en su mente que debe tener una disciplina y para ello se enlista los 14 motivos principales para lograr comenzar una startup.

Cuadro No. 4 Motivos principales para iniciar una startup

\begin{tabular}{|c|c|}
\hline 1. Networking & 2. Comprometido, \\
\hline 3. Seguridad laboral, & 4. Reconocimiento, \\
\hline 5. Hacer el bien, & $\begin{array}{l}\text { 6. Independencia } \\
\text { financiera, }\end{array}$ \\
\hline $\begin{array}{l}\text { 7. Convertirse en } \\
\text { experto, }\end{array}$ & 8. Reinvención, \\
\hline 9. Habilidades, & 10. Cambiar el mundo, \\
\hline 11. Determinación, & 12. Crear empleo, \\
\hline 13. Dinámico, & 14. Crear tu marca. \\
\hline
\end{tabular}

Fuente: Elaboración propia

\section{Recomendaciones}

Para el desarrollo de la presente propuesta es recomendable que se realice en cualquiera de los periodos semestrales, considerado por las autoridades educativas de las Instituciones Públicas de Educación Superior. Además se debe de realizar como una actividad extracurricular, a la par del currículo educativo establecido por las Instituciones Públicas de Educación Superior.

Esta propuesta está preparada para realizarse en 2 horas, durante 10 viernes y posteriormente el seguimiento hasta que se ponga en marcha la Empresa Socialmente Responsable. 


\section{Referencias:}

1. Ezequiel, A. (1998). Introducción a la planeación. España. Siglo XXI de España General.

2. Beatriz, B. (2010). Motivación inteligente. Madrid: Prentice Hal.

3, La Nueva Gestión del Talento. Construyendo compromiso. Editorial Prentice Hall 2008.

4. Libro verde: El espíritu empresarial en Europa (2003)

\section{Internet:}

\section{Revista Entrepreneur}

www.entrepreneur.com/article/267092

Pro-México http://www.promexico.gob.mx/desarrollosustentable/medio-ambiente-y-desarrollo-sustentableson-los-objetivos-principales-de-green-solutions.html

Universidad autónoma de México http://www.economia.unam.mx/publicaciones/econinfor ma/pdfs/366/08consuelo.pdf

Servicio de información sobre responsabilidad social http://www.expoknews.com/llega-la-responsabilidadsocial-a-china/ 